\title{
Do Noisy Data Exacerbate Cyclical Volatility?
}

\author{
Antulio N. Bomfim* \\ Federal Reserve Board
}

June 1999

\begin{abstract}
How does the additional uncertainty associated with noisy economic data affect business cycle fluctuations? I use a simple variant of the neoclassical growth model to show that the answer depends crucially on the assumed expectation-formation capabilities of agents. Under efficient signal extracting, noisy economic indicators dampen cyclical volatility. The opposite occurs when agents follow a simple bounded rational strategy.
\end{abstract}

JEL Classification: E32, D84, C61

Keywords: volatility, measurement error, signal extraction, expectations, bounded rationality

* The opinions expressed here are not necessarily shared by the Board of Governors of the Federal Reserve System or any members of its staff. Correspondence: Division of Monetary Affairs, Mail Stop 74, Federal Reserve Board, Washington DC 20551, USA; Tel.: (202) 736-5619; Fax: (202)452-2301; E-mail: abomfim@frb.gov. 


\section{Introduction}

This paper takes a well known microeconomic fact and analyzes its commonly overlooked macroeconomic consequences. The fact is that agents often have to make decisions on the basis of preliminary and noisy information about the world, such as the news provided by early estimates of key economic indicators. ${ }^{1}$ How does this additional layer of uncertainty at the micro level affect macroeconomic fluctuations? ${ }^{2}$ I will focus here on the relationship between noisy data and cyclical volatility. Does the added uncertainty associated with noisy data make the economy more or less volatile? Would the introduction of better economic indicators lead to dampened or wider business cycle oscillations?

The results are presented in a series of richer analyses. Section 2 introduces the basic modeling framework: a simple variant of the neoclassical growth model augmented with stochastic productivity shocks. Section 3 completes the discussion of the model by solving it under the assumption that the productivity shocks are perfectly observable. This information structure is changed in section 4 . There I assume that the state of productivity becomes observable only after production takes place. Until then, all that the agents see is a noisy indicator of the true state of productivity. I then solve the model under two polar characterizations of the agents' information processing capabilities. If we assume that agents use optimal signal extraction techniques, it can be shown that the noisy component of the indicator works to dampen cyclical volatility. In particular, the variance of output in the noisy-indicator economy is less than that associated with the economy de-

\footnotetext{
${ }^{1} \mathrm{~A}$ number of statistical studies have documented the large degree of measurement error and low signal-to-noise ratios of many economic indicators. See, e.g., Diebold and Rudebusch (1991), Mankiw, Runkle and Shapiro (1984), and Kennedy (1993).

${ }^{2}$ Other papers that have examined the macroeconomic implications of noisy indicators include Oh and Waldman $(1990,1995)$ and Kasa (1996).
} 
scribed in section 3. In contrast, if we postulate that individual behavior is characterized by a simple bounded rationality strategy, where the agents do not engage in signal extraction, then the noisy-indicator economy displays higher volatility than its perfect-indicator counterpart. Section 5 provides additional discussion of the main results and concludes.

\section{The Model}

The theoretical framework is a variant of the well-known dynamic general equilibrium model of King, Plosser and Rebelo (1988). As shown below, I make the simplifying assumption that the labor supply schedule is inelastic. ${ }^{3}$

\subsection{The Production Function}

Individual output $\left(y_{t}\right)$ is a function of capital input $\left(k_{t}\right)$ and the state of productivity $\left(A_{t}\right)$.

$$
y_{t}=\exp \left(A_{t}\right) k_{t}^{\theta}
$$

where the state of technology is subject to stochastic shocks to be described in section 3.

\subsection{Evolution of the Capital Stock}

Output not consumed constitutes gross investment, $i_{t}$. With $k_{t}$ representing the capital stock at the beginning of period $t$, and assuming that this stock depreciates at the rate $\delta, 0 \leq \delta<1$,

$$
k_{t+1}=(1-\delta) k_{t}+i_{t}
$$

\footnotetext{
${ }^{3}$ This assumption is made for simplicity only and does not affect the main conclusions.
} 


\subsection{Preferences}

Momentary utility is logarithmic in consumption $\left(c_{t}\right)$ :

$$
u\left(c_{t}\right)=\log \left(c_{t}\right)
$$

\subsection{Individual Behavior}

All agents are infinitely lived, forward looking, and discount the future at the rate $\beta$. Subject to the goods constraints,

$$
c_{t}+i_{t} \leq y_{t}
$$

as well as to conventional initial and transversality conditions, agents maximize expected utility over an infinite horizon. Abstracting from uncertainty, the objective function can be written as:

$$
\sum_{t=0}^{\infty} \beta^{t}\left\{u\left(c_{t}\right)+\lambda_{t}\left[z_{t} k_{t}^{\theta}-c_{t}-k_{t+1}+(1-\delta) k_{t}\right]\right\}
$$

where $\lambda_{t}$ is the discounted Lagrange multiplier and $z_{t} \equiv \exp \left(A_{t}\right)$.

Given the stochastic sequence $\left\{A_{t}\right\}_{t=0}^{\infty}$, as well as a particular expectationformation mechanism, the values of $\left\{c_{t}, i_{t}, \lambda_{t}\right\}_{t=0}^{\infty}$ that maximize the expected value of (5) characterize the business cycles of this artificial economy.

Setting expectational issues aside for the moment, the derivation of the system of Euler equations that corresponds to the maximization of (5) is straightforward. This system can be written as follows:

$$
\begin{aligned}
u_{c}\left(c_{t}\right)-\lambda_{t} & =0 \\
\beta \lambda_{t+1}\left[\theta y_{t+1} / k_{t+1}+(1-\delta)\right]-\lambda_{t} & =0 \\
y_{t}-c_{t}-k_{t+1}+(1-\delta) k_{t} & =0
\end{aligned}
$$


where $u_{c}\left(c_{t}\right)$ denotes the marginal utility of consumption.

\section{Decision Rules with Noiseless Data}

Log-linearizing of the Euler equations and expressing all variables in percentage deviations from the steady state lead to the following approximation to the equilibrium law of motion of the capital stock:

$$
\hat{k}_{t+1}=m_{k k} \hat{k}_{t}+m_{k l} \sum_{j=0}^{\infty} \mu^{j}\left(s_{1} A_{t+j+1}+s_{2} A_{t+j}\right)+R A_{t+1}+Q A_{t}
$$

where a "caret" over a symbol denotes that the variable is expressed in percentage deviations from the steady state (e.g., $\left.\hat{k}_{t} \equiv \log \left(k_{t} / \bar{k}\right)\right)$. The coefficients $m_{k k}, m_{k l}, s_{1}, s_{2}, R$, and $Q$, as well as the $\mu$ parameter, are all functions of the structural parameters introduced above. ${ }^{4}$

To complete the solution to the model we need two additional assumptions. The first concerns the law of motion of $A_{t}$, which I assume to follow a first-order autoregression

$$
A_{t}=\rho A_{t-1}+a_{t}
$$

where $|\rho| \leq 1$, and $a_{t}$ is white noise with variance $\sigma_{a a}$.

The second remaining assumption deals with the information structure. In particular, I start by assuming that (i) agents know the stochastic process governing $A_{t}$ and (ii) $A_{t}$ is perfectly observable at the beginning of each period. These assumptions allow us to write the capital accumulation decision rule as:

$$
\hat{k}_{t+1}=m_{k k} \hat{k}_{t}+m_{k a} A_{t}
$$

\footnotetext{
${ }^{4}$ The derivation of (9) follows King et al. (1990) and Blanchard and Kahn(1980) very closely.
} 
where

$$
m_{k a} \equiv \frac{m_{k l}\left(s_{1} \rho+s_{2}\right)}{(1-\mu \rho)}+R \rho+Q
$$

is a positive constant and $0 \leq m_{k k} \leq 1$.

Equation (11) characterizes the evolution of capital in the stochastic economy with no noisy indicator. Substitution into the (log-linearized) production function yields the equilibrium process for output:

$$
\hat{y}_{t}=A_{t}+\theta m_{k a} A_{t-1}+\theta m_{k k} \hat{k}_{t-1}
$$

Given equations (11) and (12), we can express our measure of cyclical volatility as

$$
\sigma_{y y}^{(P I)}=\left[1+\frac{\theta^{2} m_{k a}^{2}\left(1+m_{k k} \rho\right)}{\left(1-m_{k k}^{2}\right)\left(1-m_{k k} \rho\right)}+\frac{2 \theta m_{k a} \rho}{1-m_{k k} \rho}\right] \frac{\sigma_{a a}}{1-\rho^{2}}
$$

where $\sigma_{y y}^{(P I)}$ denotes the variance of output in the "perfect-indicator" economy. As shown in the above equation, the only source of output volatility in the perfect-indicator economy is the variance of the exogenous productivity shock.

\section{Decision Rules with Noisy Data}

I will now modify the information structure by assuming that agents do not observe the state of productivity before production takes place. Instead, at the beginning of each period, agents observe a preliminary announcement of the current state of technology. This preliminary announcement $\left(\pi_{t}\right)$ is subject to measurement error $\left(e_{t}\right)$ :

$$
\pi_{t}=A_{t}+e_{t}
$$


where $e_{t}$ is white noise with variance $\sigma_{e e}$ and is uncorrelated with $a_{t}$.

As in Kydland and Prescott (1982), agents follow a two-stage decision process. In the first stage, they make their factor allocation decision, which is based on the preliminary announcement. Once production takes place, the second stage begins. The representative agent can use its knowledge of output and inputs to deduce the value of the productivity shock $\left(A_{t}\right)$. Given this larger information set, agents update their forecasts of future economic conditions.

We are now ready to address the main question posed in this paper. What happens to cyclical volatility when there is noise in the indicator? In particular, how is the variance of output affected by the noisy indicator? As I show below, the answer depends on how agents deal with the measurement error problem.

\subsection{Efficient Signal Extraction}

The agents' problem is to come up with a forecast of $A_{t}$ given its noisy indicator. We start by assuming that they are endowed with a statistically efficient signal-extraction technique. In particular, equations (10) and (14) form a state-space system that can be passed through the Kalman filter to generate optimal forecasts of $A_{t}$ conditioned on the preliminary announcement $\pi_{t}$. Letting $E\left[A_{t} \mid A_{t-1}, \pi_{t}\right]$ denote this prediction, we can write

$$
E\left[A_{t} \mid A_{t-1}, \pi_{t}\right]=(1-\zeta) \rho A_{t-1}+\zeta \pi_{t}
$$

where $\zeta \equiv \sigma_{a a} /\left(\sigma_{a a}+\sigma_{e e}\right)$. Equation (15) is straightforward to derive and has a very intuitive interpretation. Accordingly, the higher the signal-to-

noise ratio $\left(\sigma_{a a} / \sigma_{e e}\right)$ associated with $\pi_{t}$, the more weight will be attached to the preliminary announcement when forming expectations of $A_{t}$. 
Again, substituting the prediction formula for $A_{t}$ into the equilibrium law of motion for $\hat{k}_{t}$ - equation (9)—yields the capital accumulation decision rule:

$$
\hat{k}_{t+1}=m_{k k} \hat{k}_{t}+m_{k a 1} A_{t-1}+m_{k \pi} \pi_{t}
$$

where $m_{k a 1} \equiv m_{k a}(1-\zeta) \rho$ and $m_{k \pi} \equiv m_{k a} \zeta$.

Let us now turn to the derivation of the variance of output, which can be computed by combining equation (16) and the log-linearized production function:

$$
\begin{array}{r}
\sigma_{y y}^{(N I E)}=\left[1+\frac{\theta^{2} m_{k a}^{2}\left(1+m_{k k} \rho\right) \rho^{2}+\left(\zeta+(2-\zeta) m_{k k} \rho\right) \zeta\left(1-\rho^{2}\right)}{\left(1-m_{k k}^{2}\right)\left(1-m_{k k} \rho\right)}+\right. \\
\left.\frac{2 \theta m_{k a} \rho\left(\rho^{2}+\zeta\left(1-\rho^{2}\right)\right)}{1-\rho m_{k k}}\right] \frac{\sigma_{a a}}{1-\rho^{2}}+\frac{m_{k a}^{2} \zeta^{2} \sigma_{e e}}{1-m_{k k}}
\end{array}
$$

where $\sigma_{y y}$ denotes the variance of output in the "noisy-indicator" economy under efficient signal processing.

The above equation has two noteworthy features. First, unlike the perfectindicator economy, there are 2 sources of output fluctuations in the noisyindicator economy: the productivity shock - which was the sole source of volatility in the PI economy - and the indicator noise. Second, it is easy to show that, for $\sigma_{e e}=0$, the equation reduces to (13). What happens to output volatility in the more general case where $\sigma_{e e} \neq 0$ ? After some tedious algebra it can be shown that

$$
\sigma_{y y}^{(P I)}-\sigma_{y y}^{(N I E)}=\frac{\left(1+m_{k k} \sigma_{e e} \zeta+\left(1-m_{k k}^{2}\right) m_{k a} \rho \sigma_{a a}(1-\zeta)\right.}{\left(1-m_{k k}^{2}\right)\left(1-m_{k k} \rho\right)}
$$

which, given that $0<\zeta<1$, implies that output is more volatile in the perfect indicator economy. Thus, even though the noisy component of the productivity indicator is an additional source of volatility, output fluctuations 
are dampened by the presence of the noisy indicator.

The intuition for this result is simple. The agents know that the indicator is contaminated by noise and use an efficient technique to estimate its signal component. Effectively, they end up basing their decisions on an efficient estimate of the state of productivity, an estimate that is necessarily smoother than the state of productivity itself. Hence, despite the additional uncertainty represented by the noise component, output is less variable than what otherwise would be the case.

\subsection{No Signal Extraction}

As shown above, $\pi_{t}$ is an unbiased indicator of the true state of productivity. What if the agents take the indicator at face value? ${ }^{5}$ In this case we can write the variance of output as

$$
\sigma_{y y}^{(N I N)}=\sigma_{y y}^{(P I)}+\frac{m_{k a}^{2} \sigma_{e e}^{2}}{1-m_{k k}}
$$

where $\sigma_{y y}^{(N I N)}$ is the variance of output in the noisy-indicator economy with no signal extraction. Equation (18) makes it clear that, for $\sigma_{e e}>0$, the noisy indicator increases output volatility when agents fail to signal-extract, a result that is in stark contrast to the efficient signal extraction case. Again, the intuition is very clear: by taking the indicator at face value, the agents end up reacting not just to fundamental shocks to productivity, but also to the pure noise associated with measurement error in the preliminary announcement.

\footnotetext{
${ }^{5}$ We can think of this behavior as a bounded rationality strategy where either the agents are unaware of the measurement error problem or they find it too costly to engage in signal extraction behavior.
} 


\section{Concluding Remarks}

The aggregate effect of noisy economic indicators depends on the degree of sophistication with which agents process incoming economic data. Noisy data dampen cyclical volatility when agents use efficient signal extraction techniques, but exacerbate it when they take the data at face value. Which of the two scenarios is more likely in the real world? This is an empirical question that goes beyond the scope of this paper and touches at the heart of the question of just how "rational" the expectations of economic agents are. Nevertheless, the results presented in paper do suggest a novel way of addressing this question. Namely, if we can identify two time periods-one with superior data, the other more prone to measurement error problemsthe one with better economic indicators should have lower cyclical volatility, other things being equal, if the agents violate the rational expectations assumption.

The results also pave the way to other avenues for future research. I am currently investigating two issues. The first relates to the quantitative importance of measurement error in business cycle fluctuations. Are the results presented here economically important? I plan to analyze this question in the context of a richer model that allows for quantitative experiments. A second direction for future research pertains to the relationship between financial market volatility and preliminary announcements of macroeconomic and business data. Is this relationship consistent with the efficient market hypothesis? The findings reported in this paper suggest that, controlling for other sources of fluctuations, the efficient market hypothesis implies that periods of decreased uncertainty about the state of the economy should coincide with bouts of higher financial market volatility. 


\section{References}

Blanchard, O. and C. Kahn, "The Solution of Linear Difference Models under Rational Expectations," Econometrica, 1980, 48, 1305-1311.

Diebold, F. and G. Rudebusch, "Forecasting Output with the Composite Leading Index: A real-time analysis," Journal of the American Statistical Association, 1991, 86, 603-610.

Kasa, K., "Signal Extraction and the Propagation of Business Cycles," 1996. Research Department, Federal Reserve Bank of San Francisco.

Kennedy, J., "An Analysis of Revisions to the Industrial Production Index," Applied Economics, 1993, 25, 213-219.

King, R., C. Plosser, and S. Rebelo, "Production, Growth and Business Cycles: 1. The Basic Neoclassical Model," Journal of Monetary Economics, 1988, 21, 195-232.

$\ldots$ _ _ _ and _ _ , "Production, Growth and Business Cycles: Technical Appendix," 1990. Rochester Center for Economic Research, University of Rochester.

Kydland, F. and E. Prescott, "Time to Build and Aggregate Fluctuations," Econometrica, 1982, 50, 1345-70.

Mankiw, N. G., D. E. Runkle, and M. D. Shapiro, "Are preliminary announcements of the money stock rational forecasts?," Journal of Monetary Economics, 1984, 14, 15-27.

Oh, S. and M. Waldman, "The Macroeconomic Effects of False Announcements," Quarterly Journal of Economics, 1990, 105, 1015-34. 
_ and _ _ , "The U.S. Government's Index of Leading Economic Indicators as a Source of Expectational Shocks," 1995. Working Paper, Cornell University. 